\title{
Origin of the Fermi arcs in cuprates: a dual role of quasiparticle and pair excitations
}

\author{
William Sacks $^{1} \oplus$, Alain Mauger ${ }^{1}$ and Yves Noat $^{2}$ \\ 1 Sorbonne Université, Institut de Minéralogie, de Physique des Matériaux et de Cosmochimie, CNRS, \\ UMR 7590, 4 Place Jussieu, 75005 Paris, France \\ 2 Sorbonne Université, Institut des Nanosciences de Paris, CNRS, UMR 7588, 4 Place Jussieu, 75005 \\ Paris, France \\ E-mail: william.sacks@ sorbonne-universite.fr
}

\begin{abstract}
Angle resolved photoemission spectroscopy (ARPES) mesurements in cuprates have given key information on the temperature and angle dependence of the gap ( $d$-wave order parameter, Fermi arcs and pseudogap). We show that these features can be understood in terms of a Bose condensation of interacting pairons (preformed hole pairs which form in their local antiferromagnetic environment). Starting from the basic properties of the pairon wavefunction, we derive the corresponding $k$-space spectral function. The latter explains the variation of the ARPES spectra as a function of temperature and angle up to $T^{*}$, the onset temperature of pairon formation. While Bose excitations dominate at the antinode, the fermion excitations dominate around the nodal direction, giving rise to the Fermi arcs at finite temperature. This dual role is the key feature distinguishing cuprate from conventional superconductivity.
\end{abstract}

Keywords: cuprates, Fermi arcs, ARPES, novel mechanisms, pseudogap

(Some figures may appear in colour only in the online journal)

\section{Introduction}

More than 30 years after their discovery by Bednorz and Müller [1], cuprates still keep their secret. The parent compounds are two-dimensional insulating antiferromagnets and superconductivity is induced by doping the $\mathrm{CuO}$ planes. The origin of the superconducting $T_{\mathrm{c}}$ dome is still unknown and the Bardeen-Cooper-Schrieffer theory (BCS) [2] fails to describe the essential properties of cuprates: the pairing and condensation mechanisms.

In addition to tunneling spectroscopy [3], angle resolved photoemission spectroscopy (ARPES) has revealed groundbreaking information on the superconducting condensate, in particular the angular dependence, which otherwise must be inferred (see for instance [4-6] and references therein). There are three major empirical findings: the $d$-wave nature of the condensate, a partial Fermi-surface at finite temperature (Fermi arcs) [7] and a spectral gap which persists at $T_{\mathrm{c}}$ in the antinodal direction up to a higher temperature $T^{*}$. The latter pseudogap [8], first found by NMR spectroscopy [9, 10], was then observed in the specific heat of $\mathrm{YBa}_{2} \mathrm{Cu}_{3} \mathrm{O}_{6+x}$ [11] and subsequently by ARPES $[12,13]$ and tunneling [14]. The relation between the pseudogap state and non-BCS superconductivity is still strongly debated.

In earlier works [7], it was suggested that the Fermi arcs seen above the critical temperature collapse to virtually a single point in $k$-space at the nodes at $T_{\mathrm{c}}$, compatible with a $d$-wave symmetry of the order parameter. However, more recent data with better resolution $[5,15,17]$ show that a finitesized Fermi arc still exists at $T_{\mathrm{c}}$ around each node. Its origin is undecided and it is unclear whether these Fermi arcs are tied to $T_{\mathrm{c}}$ or rather to $T^{*}$. Recent effort has focused on the gap function near the node (nodal gap), often proposed to be the superconducting order parameter $[15,16]$. In this context, the antinodal pseudogap is attributed to some extraneous competing order, such as a spin or charge density wave [18, 19]. On the other hand, based on ARPES measurements in the antinodal direction, Kanigel et al [20] and Shi et al [21] stressed the preformed-pair interpretation. 
In this article, we answer these questions in the framework of the condensation of preformed pairons [22]. To proceed, we calculate the spectral function for cuprates and directly compare the computed energy distribution curves (EDCs) to the ARPES measurements as a function of temperature and angle at the Fermi surface. We show that the condensation of pairons fully describes the continuous evolution of the ARPES spectra with temperature and angle, from the antinode to the node. Remarkably, the boson condensation is revealed in the antinodal (AN) direction where Bose-Einstein statistics dominate, whereas the fermion excitations dominate near the nodal (N) direction. At $T_{\mathrm{c}}$ and above, the pseudogap is revealed by the incoherent pair excitations at the antinode, which coexist with the Fermi arcs around the nodes - a direct consequence of the composite character of pairons.

\section{From pairons to Cooper pairs}

In the framework of the $t-J$ Hamiltonian, it has been shown that two holes in an antiferromagnetic system form a bound state provided that the ratio $J / t$ is sufficiently large [23, 24]. In a recent work [22], we have extended this mechanism to a more realistic system with a large number of holes. In this scenario, pairs of holes are trapped in their anti ferromagnetic environment, on the scale of the antiferromagnetic correlation length $\xi_{\mathrm{AF}}$, forming pairons. This idea is strongly supported by the experimental finding that $\xi_{\mathrm{AF}} \sim 1 / \sqrt{p}$ [25], where $p$ is the number of holes per copper atom. Thus $\xi_{\mathrm{AF}}$ varies as the distance between holes (or hole pairs), providing an immediate explanation for the linear variation of the antinodal gap, $\Delta_{p}$, with hole doping $p$ [22].

We start with a boson Hamiltonian corresponding to a gas of non-interacting pairons which, in absence of mutual interactions, describes the incoherent pseudogap state:

$$
H_{\mathrm{B}}=\sum_{i} \varepsilon_{i} b_{i}^{\dagger} b_{i}
$$

where the operator $b_{i}^{\dagger}$ creates a given boson of energy $\varepsilon_{i}$.

In the two-particle center of mass the pairon wavefunction $\psi_{i}(\vec{r})$, (where $\vec{r}=\overrightarrow{r_{1}}-\overrightarrow{r_{2}}$ is the distance between the two holes) is determined by the AF environment, which imposes its symmetry. As a result, $\psi_{i}(\vec{r})$ has to vanish along the lattice diagonal, which corresponds to the nodal direction in $k$-space (see figure 1). We thus take the wavefunction to be the generic form:

$$
\begin{aligned}
\psi(\vec{r}) & =\frac{1}{\sqrt{4}}[\varphi(\vec{r}-a \hat{x})+\varphi(\vec{r}+a \hat{x}) \\
& -\varphi(\vec{r}-a \hat{y})-\varphi(\vec{r}+a \hat{y})],
\end{aligned}
$$

where $a$ is the lattice parameter, and $\varphi(\vec{r})=\mathrm{e}^{-\frac{r^{2}}{2 b^{2}}} / \sqrt{2 \pi b^{2}}$. The parameter $b$ fixes the spatial extension of the pairon wavefunction $\psi_{i}$ (see figure 1).

The pairon wavefunction $\psi_{i}$ and associated operator $b_{i}$ can equivalently be described by a superposition of delocalized Cooper pairs,
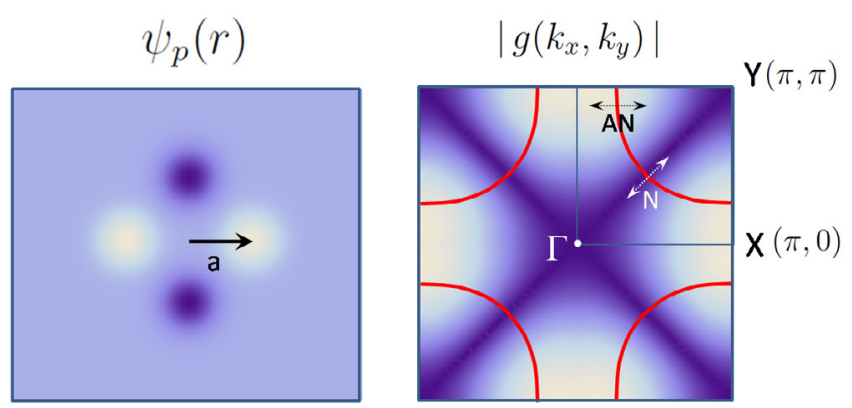

Figure 1. Pairon wavefunction (left panel) in the center of mass $(\vec{R}=0)$ and its Fourier transform (right panel). The Fermi surface, indicated by the red curve in the right panel, is calculated based on the accurate band structure proposed by Markiewicz et al [26]. In the right panel the antinodal direction $(\mathrm{AN}$ or $\theta=0)$ and the nodal direction ( $\mathrm{N}$ or $\theta=\pi / 4)$ are indicated.

$$
b_{i}=\sum_{\vec{k}} g_{\vec{k}}^{i} b_{\vec{k}}^{i}
$$

where the operator $b_{\vec{k}}^{i \dagger}=c_{\vec{k}}^{i \dagger} c_{-\vec{k}}^{i \dagger}$ creates a Cooper pair $|\vec{k} \uparrow,-\vec{k} \downarrow\rangle$. In this formulation, just as in the original Cooperpair problem [2], the ground state of the system is constructed from pairs in the zero-momentum state. The weight $g_{\vec{k}}$ appearing in the sum is given by the Fourier transform of the wavefunction $g^{i}\left(k_{x}, k_{y}\right)=\int \mathrm{e}^{\mathrm{i} \vec{k} \cdot \vec{r}} \psi_{i}(\vec{r}) \mathrm{d}^{2} \vec{r}$.

Taking the quantum average $b_{i}^{+} b_{i} \approx\left\langle b_{i}^{+}\right\rangle b_{i}+b_{i}^{+}\left\langle b_{i}\right\rangle$, and using the standard BCS expression $\left\langle b_{i}\right\rangle=\frac{\Delta_{k}^{i}}{2 E_{k}^{i}}$ we obtain the mean-field Hamiltonian:

$$
H_{\mathrm{MF}}=\sum_{\vec{k}, i} \epsilon_{\vec{k}} c_{\vec{k}}^{i+} c_{\vec{k}}^{i}+\sum_{i, \vec{k}} \Delta_{\vec{k}}^{i} b_{\vec{k}}^{i+}+\sum_{i, \vec{k}} \Delta_{\vec{k}}^{i *} b_{\vec{k}}^{i},
$$

where the first term is the kinetic energy and the second is the pairing term. In the latter, the binding energy $\Delta_{k}^{i}$ is determined by the self-consistent equation

$$
\Delta_{\vec{k}}^{i}=\varepsilon_{i} \sum_{\vec{k}^{\prime}} g_{\vec{k}}^{i} g_{\vec{k}^{\prime}}^{i}\left(\frac{\Delta_{\vec{k}^{\prime}}^{i}}{2 E_{\vec{k}^{\prime}}^{i}}\right)
$$

In the continuum limit the sum is replaced by an integral in the standard fashion. The latter expression bears a strong analogy with the BCS gap equation, however both the integration limits and the integrand involving $g_{\vec{k}}$ differ quantitatively.

Dropping the $i$ index, one can show that $g_{\vec{k}}$ takes the form:

$$
g_{\vec{k}} \propto \mathrm{e}^{-k^{2} b^{2} / 2}\left[\cos \left(k_{x} a\right)-\cos \left(k_{y} a\right)\right],
$$

which reveals both the extent of the $k$-states involved, and $g_{k_{\mathrm{F}}} \propto \cos (2 \theta)$ imposes the $d$-wave dependence of the gap parameter. Note that equations (4) and (5) imply the existence of quasiparticles of the form:

$$
E_{\vec{k}}^{i}=\sqrt{\epsilon_{\vec{k}}^{2}+\Delta_{\vec{k}}^{i^{2}}}
$$

associated with degenerate pairons of binding energy $\varepsilon_{i}$. 


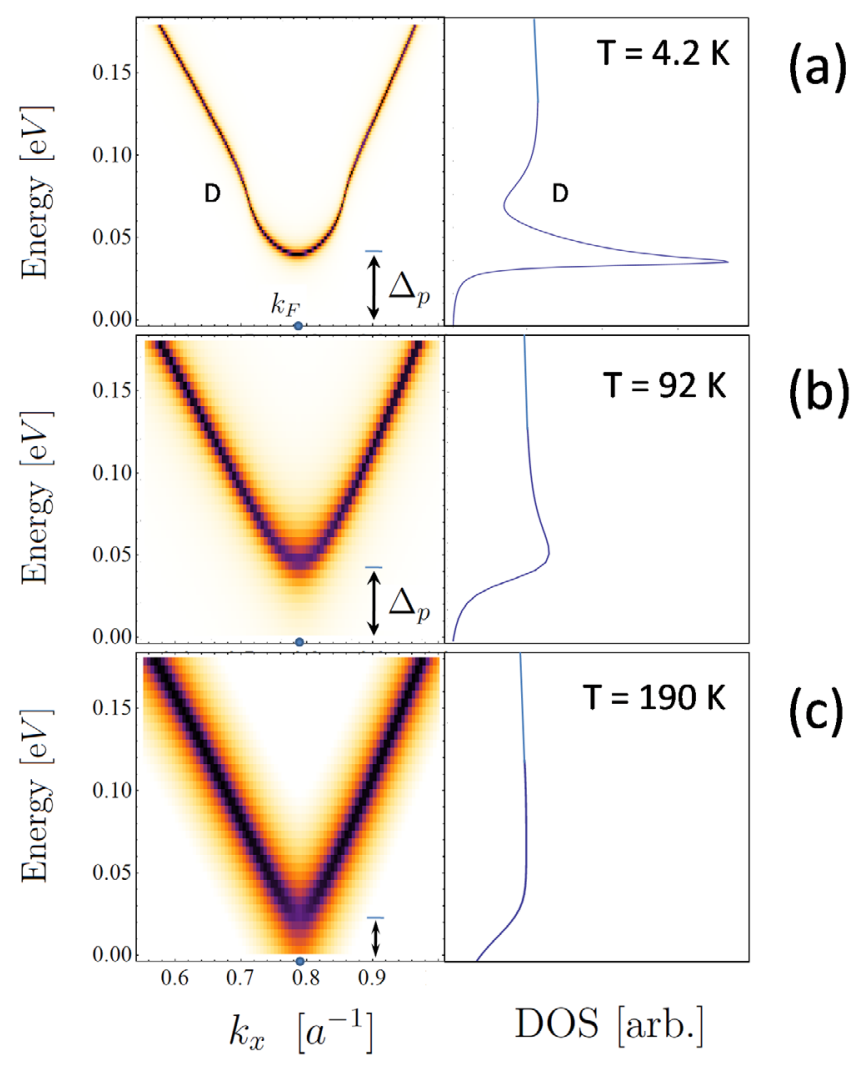

Figure 2. Spectral function $A(\vec{k}, E)$ and associated DOS along the antinodal direction at low temperature ((a), $T=4.2 \mathrm{~K})$, at the critical temperature ((b), $T=92 \mathrm{~K})$ and close to $T^{*}((\mathrm{c}), T=190$ $\mathrm{K})$, with the broadening parameter $\Gamma$ in equation (15) given by $\Gamma=k_{\mathrm{B}} T$. Note that we plot here the excited-state spectral function where the coherence factors are absent (see text).

Let us emphasize that the $i$-index is formally equivalent to a band index in the context of multi-band superconductivity [27]. However in our case, the $i$-index represents pairons of differing binding energies and the Hamiltonian (4) represents a non-superconducting state of independent Cooper pairs. In order to establish a macroscopic coherent state, a coupling between pairs of different energies is necessary [28-30]. Without the interaction term between pairons, no long range order is possible. Still, a gap in the density of states is present at the Fermi level without the characteristic peaks indicating phase coherence, see figure 2(b). Thus the Hamiltonian (4) provides a description of the main features of the pseudogap state.

Our numerical study of the self-consistent equation (5) shows that, for values of $\varepsilon_{i}$ in the relevant range for cuprates $\left(\varepsilon_{i} \sim 80-210 \mathrm{meV}\right), \Delta_{\vec{k}}^{i}$ can be well approximated by

$$
\Delta_{\vec{k}}^{i}=c \varepsilon_{i} g_{\vec{k}}
$$

where $c$ is a constant. Taking the parameter $b=a / 4$ in the calculation, we find $c \simeq 0.25$ and an energy gap $\sim 40 \mathrm{meV}$ at optimal doping. The satisfying conclusion is that the Cooperpair binding energy $\Delta_{\vec{k}}^{i}$ is proportional to the pairon energy $\varepsilon_{i}$, and thus both concepts are formally equivalent.

In the absence of pairon-pairon interactions, the system is in an incoherent state, the 'Cooper glass' [28-30]. The pairon energies are distributed with a pair density of states $P_{0}\left(\Delta_{i}\right)$, characterized by the mean value $\Delta_{0}$ and dispersion $\sigma_{0}$. A convenient form is the Lorentzian:

$$
P_{0}\left(\Delta_{i}\right) \propto \frac{\sigma_{0}^{2}}{\left(\Delta_{i}-\Delta_{0}\right)^{2}+\sigma_{0}^{2}} .
$$

As will be revealed in the spectral function, equations (4)-(8) describe the non-superconducting incoherent state giving rise to the pseudogap phenomena at the critical temperature and above.

\section{Pairon condensation}

As a result of the interaction between pairons, described by the additional coupling term

$$
H_{\mathrm{int}}=\sum_{i \neq j} V_{i j} b_{i}^{\dagger} b_{j},
$$

the system condenses in a homogeneous ground state where pairons lie in the same quantum state. All Cooper pairs are then characterized by a unique binding energy $\Delta^{i}=\Delta_{p}$. In the mean field approximation, $\Delta_{p}$ is given by a self-consistent equation, which in the antinodal direction has the form [28]:

$$
\Delta_{p}=\Delta_{0}-2 \beta P_{0}\left(\Delta_{p}\right)
$$

It includes a pair-field term proportional to the average interaction energy $\beta$. In previous work we showed that this interaction follows the critical dome and has the value $\beta \sim 2 k_{\mathrm{B}} T_{\mathrm{c}}$. Moreover, the pairon condensate model matches the phase diagram for a wide range of doping in terms of a single energy scale, $J$, the exchange energy [22].

Using the gap equation (9), and the energy bands $\epsilon_{k}$ of cuprates from Markiewicz et al [26], the spectral function of quasiparticles is obtained for any wavevector $\vec{k}$. Assuming nearly optimal doping $\left(\Delta_{p}(0)=40 \mathrm{meV}, T_{\mathrm{c}}=92 \mathrm{~K}\right)$ the spectral intensity in the AN direction crossing $k_{\mathrm{F}}$ is shown in figure 2 (left panels) at three different temperatures. The $k$ sum of these spectra gives directly the associated quasiparticle density of states (DOS) relevant to tunneling (right panel).

A well-defined gap is visible at low temperature with a strong change of slope above the gap energy $\Delta_{p}$ giving rise to the characteristic dip in the DOS (a), which has been widely observed by tunneling spectroscopy in cuprates (see [3] and references therein). At the critical temperature (b), a pseudogap persists in the DOS which finally disappears at a much higher temperature $T^{*}$ (c). In the remainder of this work we focus on the EDCs with the wave vector on the Fermi surface to compare with the experiments.

\section{Pair densities}

We start with the low-temperature SC state where all pairs belong to the condensate. An essential concept of the model is that, upon rising temperature, pairs are excited out of the condensate without pair-breaking - a highly non-BCS feature. 


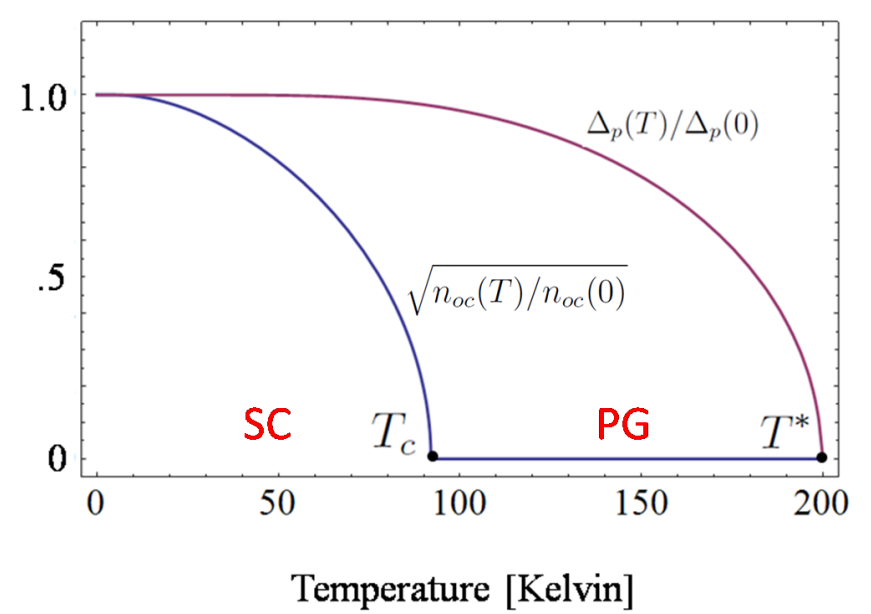

Figure 3. Temperature phase diagram in the pairon model for optimal doping: critical curves corresponding to $\sqrt{n_{\mathrm{oc}}(T)}$ (lower curve) and the antinodal gap $\Delta_{p}(T)$ (upper curve). As indicated, the condensate density vanishes at $T_{\mathrm{c}}$, while pairs exist up to $T^{*}$.

Taking the relevant Bose-Einstein statistics with $\mu=0$, and assuming $d$-wave pairing, the condensate angular density is given by:

$$
\begin{aligned}
n_{\mathrm{oc}}(T, \theta)= & n_{0}-\mathcal{A}(T, \theta) \int_{\Delta_{p} \cos (2 \theta)+\delta}^{\infty} \mathrm{d} \Delta_{i} P_{0}\left(\Delta_{i}\right) \\
& \times f_{\mathrm{B}}\left(\Delta_{i}-\Delta_{p} \cos (2 \theta), T\right),
\end{aligned}
$$

where $f_{\mathrm{B}}(E, T)=\left(\mathrm{e}^{\frac{E}{k_{\mathrm{B}} T}}-1\right)^{-1}$ is the Bose-Einstein distribution, $\delta$ is a low-energy cut-off [28] and $\mathcal{A}(T, \theta)$ is a normalization factor to be discussed below. The constant $n_{0}$, directly proportional to the doping value, is assumed to be independent of $\theta$.

The integrated condensate density, $\int \mathrm{d} \theta n_{\mathrm{oc}}(T, \theta) /(2 \pi)$, shown in figure 3 , is a monotonically decreasing function of temperature (lower curve), due to pair excitations, and vanishes at $T_{\mathrm{c}}$ as expected. Note that in this temperature range, the antinodal gap $\Delta_{p}(T)$ (upper curve), reflecting the total number of pairs, is practically constant up to $T_{\mathrm{c}}$, in agreement with experiment. However for higher temperatures, $T>T_{\mathrm{c}}$ the gap $\Delta_{p}(T)$ markedly decreases to finally vanish at $T^{*}$ as a result of pair dissociation [31].

The normalization factor $\mathcal{A}(T, \theta)$ is determined using appropriate boundary conditions. We assume that, even at finite temperature (below $T_{\mathrm{c}}$ ), the condensate density remains uniform: $n_{\mathrm{oc}}(T, \theta)=n_{\mathrm{oc}}(T)$. Furthermore, the temperature dependence of the antinodal gap $\Delta_{p}(T)$ (figure 3) is taken throughout this work as the BCS function, however with the ratio $\Delta_{p}(0) / k_{\mathrm{B}} T^{*}=2.2$ compatible with the gap equation (5). Finally, imposing $n_{\mathrm{oc}}\left(T_{\mathrm{c}}\right)=0$, we obtain a self-consistent form for $\mathcal{A}(T, \theta)$ with the quadrature:

$$
\begin{aligned}
\mathcal{A}(T, \theta)^{-1}= & \left(1-n_{\mathrm{oc}}(T)\right)^{-1} \\
& \times \int_{\Delta_{p} \cos (2 \theta)+\delta}^{\infty} \mathrm{d} \Delta_{i} P_{0}\left(\Delta_{i}\right) \\
& \times f_{\mathrm{B}}\left(\Delta_{i}-\Delta_{p} \cos (2 \theta), T\right) .
\end{aligned}
$$

Note that the latter self-consistent equation implies the constraint of particle conservation at all temperatures.

Let us now consider the excited-pair and dissociated-pair densities. The latter dissociation phenomenon occurs if the pair binding energy is typically small compared to the thermal energy. As in BCS theory, this process is governed by the Fermi-Dirac distribution $f(E, T)=\left(\mathrm{e}^{\frac{(E-\mu)}{k_{\mathrm{B}} T}}+1\right)^{-1}$ giving rise to the $\left[1-\tanh \left(\frac{E}{k_{\mathrm{B}} T}\right)\right]$ factor in the number of dissociated pairs:

$$
\begin{aligned}
n_{\text {diss }}(T, \theta) & =\mathcal{A}(T, \theta) \int_{\Delta_{p} \cos (2 \theta)+\delta}^{\infty} \mathrm{d} \Delta_{i} P_{0}\left(\Delta_{i}\right) \\
& \times f_{\mathrm{B}}\left(\Delta_{i}-\Delta_{p} \cos (2 \theta), T\right) \\
& \times\left[1-\tanh \left(\frac{\Delta^{i}}{k T}\right)\right] .
\end{aligned}
$$

In a similar way, one can write the excited pair density:

$$
\begin{aligned}
n_{\mathrm{ex}}(T, \theta) & =\mathcal{A}(T, \theta) \int_{\Delta_{p} \cos (2 \theta)+\delta}^{\infty} \mathrm{d} \Delta_{i} P_{0}\left(\Delta_{i}\right) \\
& \times f_{\mathrm{B}}\left(\Delta_{i}-\Delta_{p} \cos (2 \theta), T\right) \\
& \times \tanh \left(\frac{\Delta^{i}}{k T}\right) .
\end{aligned}
$$

The three densities must follow the sum rule:

$$
n_{\mathrm{oc}}(T)+n_{\mathrm{ex}}(T, \theta)+n_{\mathrm{diss}}(T, \theta)=n_{0},
$$

which can be verified by inspection. Furthermore, they inherently capture the physical properties of the cuprate phase diagram, from $T=0$ through $T_{\mathrm{c}}$ up to $T^{*}$. It is remarquable that both Bose-Einstein and Fermi-Dirac statistics appear in $n_{\mathrm{ex}}$ and $n_{\text {diss }}$ - a direct consequence of the composite nature of the pairons.

\section{Spectral function}

Similarly as for the DOS [31], the spectral function $A(\vec{k}, E)$ can be expressed as a sum of three terms, the condensate spectral function $A_{\text {cond }}(\vec{k}, E)$, the excited pairs contribution $A_{\mathrm{ex}}(\vec{k}, E)$ and finally the dissociated pairs term $A_{\text {diss }}(\vec{k}, E)$. The first term is essentially determined by the number of condensed pairs with energy $\Delta_{p}$, associated with the quasiparticles $E_{\vec{k}}=\sqrt{\epsilon_{\vec{k}}^{2}+\Delta_{\vec{k}}^{2}}$, where $\Delta_{k}$ is the condensate gap function [28]:

$$
A_{\text {cond }}(\vec{k}, E)=\frac{-1}{\pi} \Im m \frac{n_{\mathrm{oc}}(T)}{E-E_{k}+\mathrm{i} \Gamma} .
$$

$\Gamma$ is the standard parameter describing energy broadening.

In the latter equation we have neglected the coherence factors for occupied versus unoccupied states, which corresponds to the spectral function for excited states (both hole and electron-type). In this case, as shown by Schrieffer [32], for symmetric bands at the Fermi level, the coherence factors 

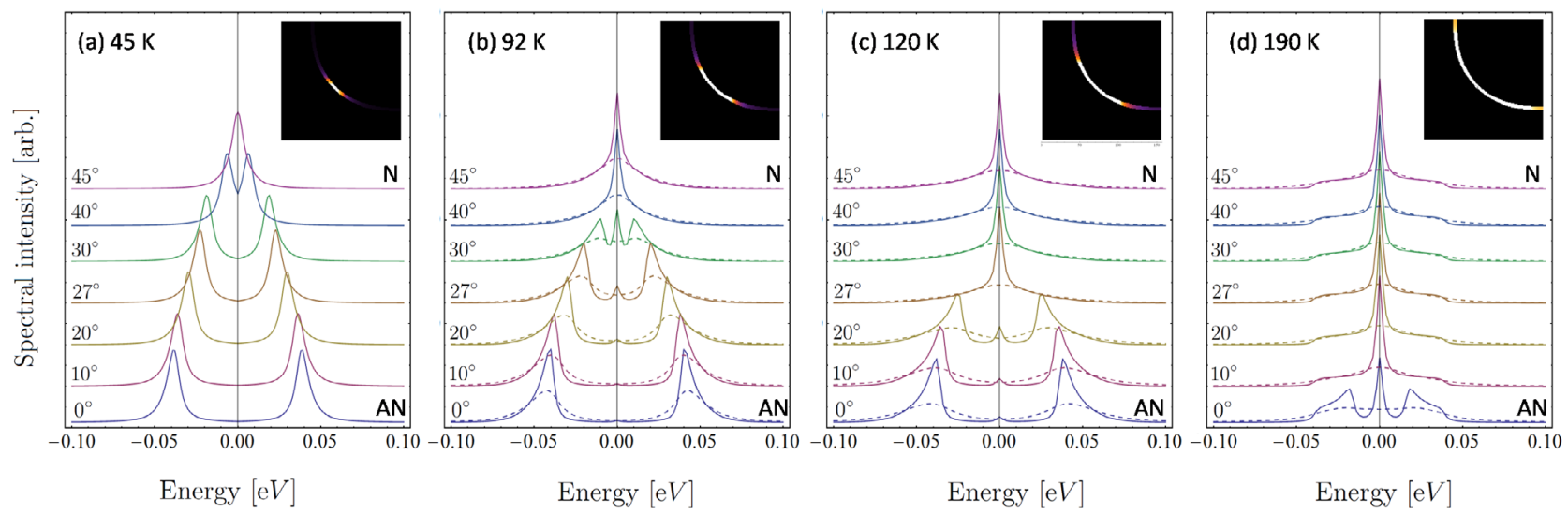

Figure 4. Spectral function plots at the Fermi energy $A\left(\overrightarrow{k_{\mathrm{F}}}, E\right)$ as a function of angle at different temperatures $T=45,92,120,190 \mathrm{~K}$. Plain lines: spectral function calculated for $\Gamma=2 \mathrm{meV}$; Dashed lines: spectral function calculated for $\Gamma=k_{\mathrm{B}} T$. Note that, contrary to a BCS picture, it is the excited pairs that dissociate giving rise to the Fermi arcs (peaks at the Fermi level). (a) 45 K. (b) 92 K. (c) 120 K. (d) $190 \mathrm{~K}$. The insets are the calculated Fermi arcs in the reduced Brillouin zone.

disappear. Since we are concerned here with energies very close to the Fermi level, where the particle/hole asymmetry is small (i.e. with no additional self-energy), equation (15) provides a good approximation for the EDCs.

The excited-pair term of the spectral function results from thermal excitations of pairons out of the condensate:

$$
\begin{aligned}
A_{\mathrm{ex}}(\vec{k}, E) & =\frac{-\mathcal{A}(T, \theta)}{\pi} \Im m \int \frac{\mathrm{d} \Delta_{i} P_{0}\left(\Delta_{i}\right)}{E-E_{\vec{k}}^{i}+\mathrm{i} \Gamma} \\
& \times f_{\mathrm{B}}\left(\Delta^{i}-\Delta_{p}(T), T\right) \tanh \left(\frac{\Delta^{i}}{k T}\right) .
\end{aligned}
$$

Finally, the dissociated pair term is caused by the thermal dissociation of Cooper pairs into normal fermions of energy $\epsilon_{\vec{k}}$. It has the simple expression:

$$
A_{\mathrm{diss}}(\vec{k}, E)=\frac{-\mathcal{A}(T, \theta)}{\pi} \Im m \frac{n_{\mathrm{diss}}(T, \theta)}{E-\epsilon_{\vec{k}}+\mathrm{i} \Gamma} .
$$

The total spectral function at the Fermi energy $A\left(\vec{k}_{\mathrm{F}}, E\right)$, comparable to the measured EDC, is shown in figure 4 as a function of angle for four different temperatures, ranging from low $T$ up to a temperature close to $T^{*}$. The corresponding Fermi surface is indicated by the inset in each panel. Two values of the broadening parameter were considered. First a small value, $\Gamma=2 \mathrm{meV}$ to highlight the intrinsic spectral weight (solid lines). Secondly, choosing $\Gamma=k_{\mathrm{B}} T$ accounts for the thermal broadening (figure 4, dashed lines) present in experiments.

At low temperature, $T=45 \mathrm{~K}$ (which is well below $T^{*} \approx 200 \mathrm{~K}$ ), two well-defined Bogoliubov peaks are clearly visible in the spectra (figure 4(a)). The latter is maximum in the AN direction, at the energy $\pm \Delta_{p}$, and decreases as a function of angle to vanish in the $\mathrm{N}$ direction in agreement with $d$-wave symmetry: $\Delta_{p} \cos (2 \theta)$. However, in the vicinity of the node, the spectra exhibit a peak at $E=0$ which originates from dissociated pairs. Thus, at low temperature the spectral function $A\left(\vec{k}_{\mathrm{F}}, E=0\right)$ is zero along the Fermi surface except around the $\mathrm{N}$ direction where a tiny arc is revealed (inset of figure 4(a)). This arc of normal states reduces to a 'Fermi point' at zero temperature, in agreement with experiments [5].

At the critical temperature (figure 4(b)), the gap closes well before the node at the critical angle $\theta_{\mathrm{c}}$ above which the peaks remain at the Fermi level. This effect constitutes the critical Fermi arc which, as will be explained below, results from pair breaking concomitant with thermally induced quasiparticle excitations. Even above $T_{\mathrm{c}}$, (c), Bogoliubov coherence peaks are still present in the AN direction, due to excited pairs in the pseudogap state. As the temperature continues to increase, the Fermi arc progressively expands and finally the full Fermi surface is almost recovered at $T=190 \mathrm{~K}$, which is close to $T^{*} \approx 200 \mathrm{~K}(\mathrm{~d})$.

\section{Comparison to ARPES experiments}

Hashimoto et al [5] have done extensive ARPES studies of slightly underdoped $\mathrm{Bi}_{2} \mathrm{Sr}_{2} \mathrm{CaCu}_{2} \mathrm{O}_{8+\delta}\left(T_{\mathrm{c}}=92 \mathrm{~K}\right)$. In figures 5 and 6 , we compare the temperature dependence of the calculated EDC spectra at the anti-node $(\theta=0)$ and near the node at the critical angle $\left(\theta_{\mathrm{c}}=31^{\circ}\right)$ to the experimental spectra reported in [5]. The overall similarity between the two sets of spectra is striking. As discussed previously, in figure 5 one observes clear Bogoliubov peaks at $\sim 40 \mathrm{meV}$ that remain virtually constant in energy up to $T_{\mathrm{c}}$ and then slowly close and vanish at the higher temperature $T^{*}$. On the contrary, at the critical angle nearer the node (figure 6) the closing of the gap, initially of smaller value $\sim 19 \mathrm{meV}$, is at $T_{\mathrm{c}}$. This Fermi arc formation is directly seen in figure 4(b). Further ARPES data confirms that the closing temperature of the Bogoliubov peaks is indeed a monotonic function of angle at the Fermi surface.

The temperature-dependent Fermi arc is thus governed by a single mechanism. Consider the natural hypothesis that each Cooper pair, as in the BCS theory [2], is subject to quasiparticle excitations as a function of temperature. Consequently, the gap amplitude $\Delta(\theta)$ for a given pair must follow the BCS-type temperature dependence and close at a temperature such that $\Delta(\theta) / k_{\mathrm{B}} T \sim C$, where $C$ is a constant of order 2 . 

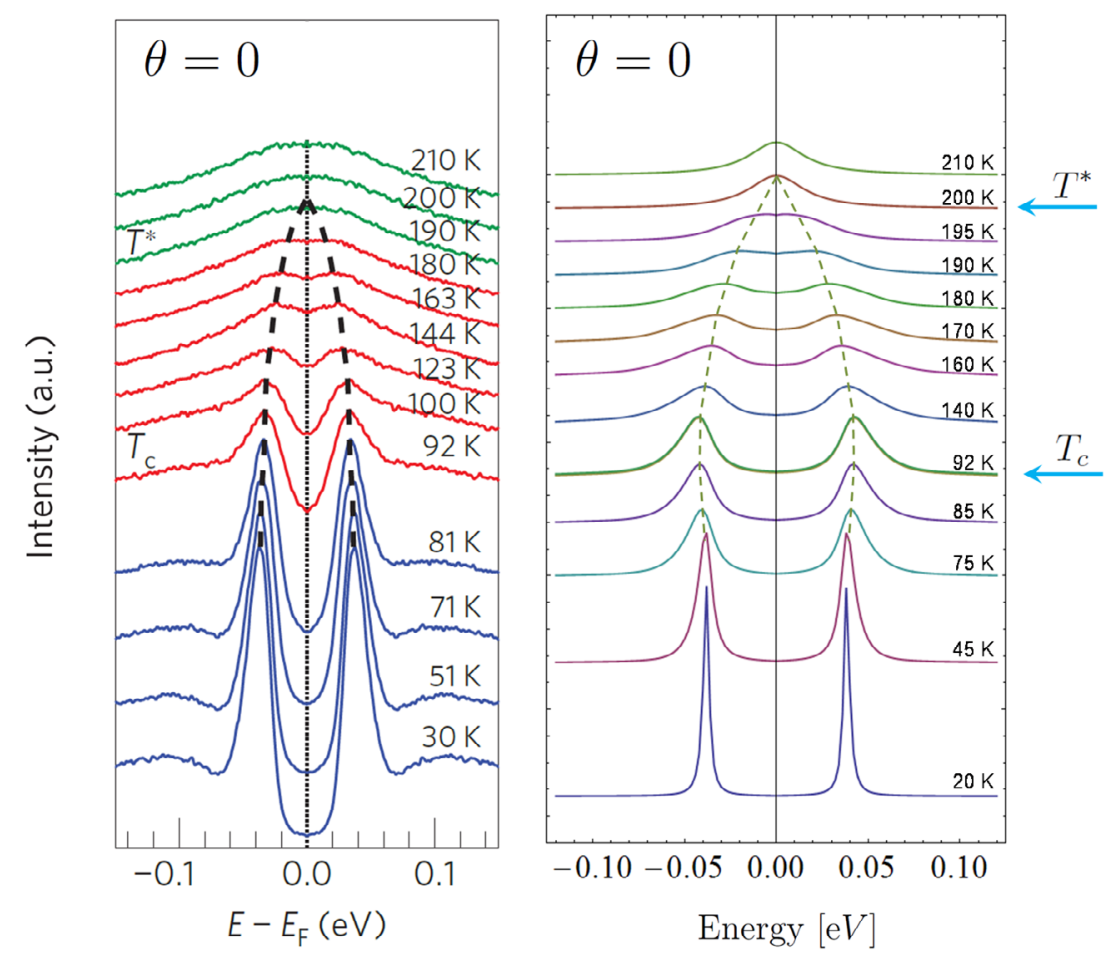

Figure 5. Left panel: measured ARPES EDC spectra (symmetrized) at the Fermi surface as a function of temperature in the antinodal direction (data from Hashimoto et al [5]). Right panel: calculated spectral function at the Fermi surface $A\left(\vec{k}_{\mathrm{F}}, E\right)$ as a function of temperature in the antinodal direction. The broadening parameter is $\Gamma=k_{\mathrm{B}} T$. Note that the incoherent background of the experimental spectrum is not accounted for. [5] (2014) (@ 2018 Springer Nature Limited. All rights reserved). With permission of Springer.
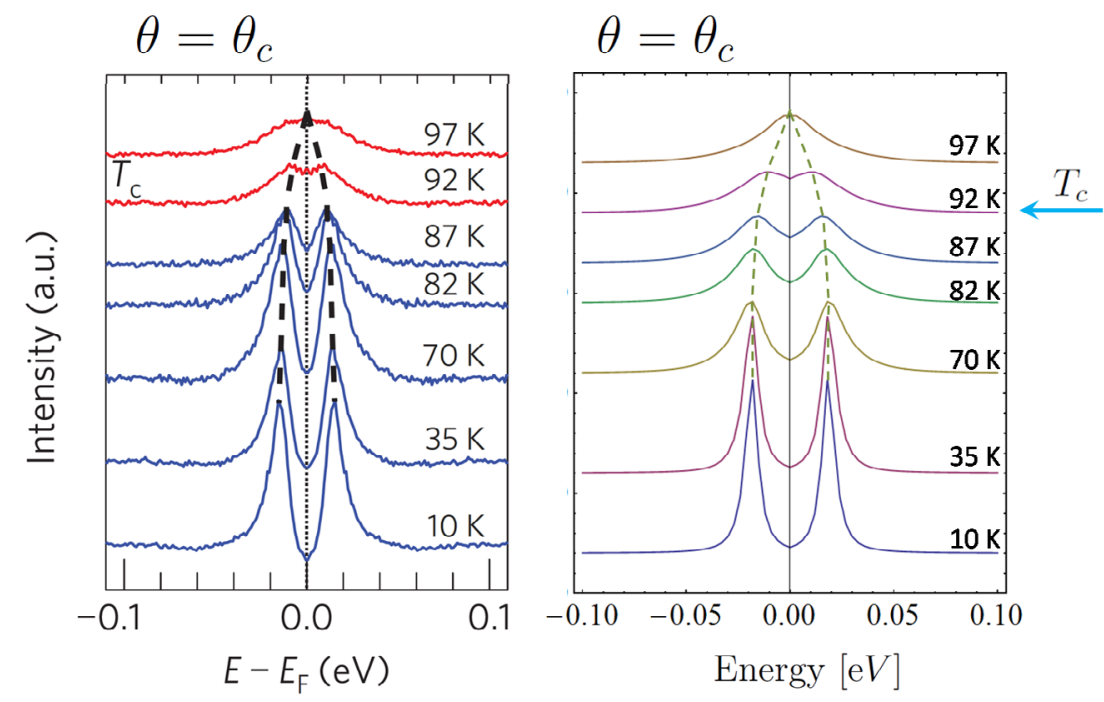

Figure 6. Left panel: measured ARPES EDC spectra (symmetrized) at the Fermi surface as a function of temperature near the node $\left(\theta=31^{\circ}\right)$ (data from Hashimoto et al [5]). Right panel: calculated spectral function at the Fermi surface $A\left(\vec{k}_{\mathrm{F}}, E\right)$ as a function of temperature near the node. The broadening parameter is $\Gamma=k_{\mathrm{B}} T$. [5] (2014) (@ 2018 Springer Nature Limited. All rights reserved). With permission of Springer.

A simple extension of the pairon gap equation (5) to finite temperature gives this result. From the available ARPES and tunneling data we find a good agreement using $C=2.2$. We note that this value is slightly larger than the BCS value, $C_{\mathrm{BCS}} \simeq 1.76$, indicating strong coupling.

The overall interpretation of the angular and temperature evolution of the ARPES EDCs is illustrated in figure 7. At zero temperature, the condensate gap $\Delta_{\vec{k}}$ has the standard $d$-wave symmetry, with the angular dependence $\cos (2 \theta)$, closing in the $\mathrm{N}$ direction, the Fermi point observed in ARPES measurements.

As the temperature rises, some pairons are excited out of the condensate. In the AN direction, their concentration $n_{\mathrm{ex}}(T, \theta=0)$ is determined by Bose statistics, however their binding energy decreases with $\theta$ due to the $d$-wave symmetry and, for a given angle, $\Delta_{p}(T, \theta)$ decreases according to the BCS function. Therefore all the excited pairons with energy less than $2.2 k_{\mathrm{B}} T$ will dissociate, leading to the Fermi arc of normal 


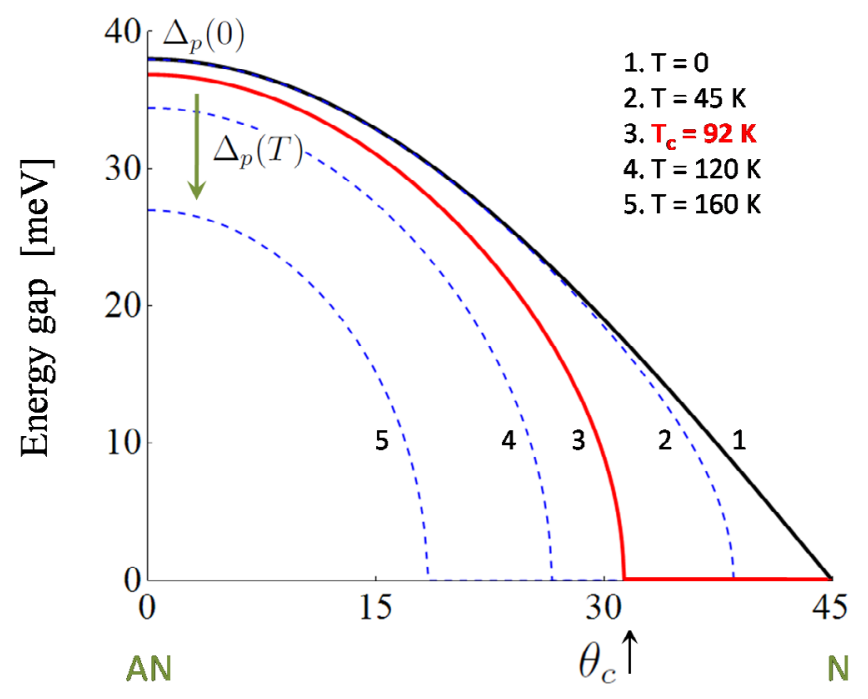

Angle [deg.]

Figure 7. Theoretical angular and temperature dependence of the gap function (Bogoliubov peak), $\Delta_{p}(T, \theta)$, illustrating the Fermiarc formation near the node $(\mathrm{N})$. For very low temperatures, only a tiny Fermi arc exists around the node and the gap is essentially $d$-wave. At $T_{\mathrm{c}}$ the Fermi arc extends to the critical angle $\theta_{\mathrm{c}}$ while the antinodal gap (AN) has hardly decreased (from underdoped to optimally doped cases). Above $T_{\mathrm{c}}$ the Fermi arcs continue to grow but now the antinodal gap decreases concomitantly.

states in the region near the node (between $45^{\circ}$ and the intercept between the $\Delta_{p}(T, \theta)$ curve with the abscissa in figure 7$)$.

We stress that this nodal pair dissociation is due to quasiparticule excitations driven by the Fermi-Dirac statistics, as given explicitly in equations (12) and (13). At the critical temperature, the Fermi arc has an angular width $\theta_{\mathrm{c}}$ to $\frac{\pi}{2}-\theta_{\mathrm{c}}$ where $\theta_{\mathrm{c}}$ satisfies the relation:

$$
\Delta_{p}(0) \times \cos \left(2 \theta_{\mathrm{c}}\right)=2.2 k_{\mathrm{B}} T_{\mathrm{c}} .
$$

While weakly bound pairs first dissociate near the node, pairs persist in the antinode and are progressively excited out of the condensate up to the critical temperature where $\Delta_{p}\left(T_{\mathrm{c}}, \theta=0\right)$ has hardly decreased and where $n_{\mathrm{oc}}\left(T_{\mathrm{c}}\right)=0$ (see figure 7 , red curve). Note that the pairons in the condensate do not directly contribute to the Fermi arcs, the consequence of our assumption that their concentration $n_{\mathrm{oc}}(T)$ is independent of angle.

Above $T_{\mathrm{c}}$, as the arc continues to extend away from the node, the gap progressively closes in the antinode as more and more pairs are being dissociated into quasiparticles. Finally, all pairs are dissociated near $T^{*}$, where the full Fermi surface is recovered. As discussed previously, this temperature is given empirically by the relation:

$$
\Delta_{p}(0)=2.2 k_{\mathrm{B}} T^{*},
$$

regardless of the doping. Therefore, using (18), we have the important relation

$$
\cos \left(2 \theta_{\mathrm{c}}\right) \simeq \frac{T_{\mathrm{c}}}{T^{*}} .
$$

This equation should be valid for all doping values in the phase diagram. Thus the critical angle should satisfy: $\cos \left(2 \theta_{c}\right)=f(p)$, where $f(p)$ is a unique function of the carrier concentration.

In the underdoped limit $\left(T^{*} \gg T_{\mathrm{c}}\right)$ the critical angle is close to $\pi / 4$ indicating very small nodal Fermi arcs at $T_{\mathrm{c}}$. Then the antinodal boson character dominates the phase transition. To the contrary, on the overdoped side $\left(T^{*} \sim T_{\mathrm{c}}\right)$ the Fermi arcs grow very rapidly with temperature indicating the coexistence of fermion and boson excitations, in good agreement with experiments $[15,17]$. Thus, the cuprate phenomenology is in fact continuous as a function of doping while the simple relation (20) implies that the superconducting order, the pseudogap state and the Fermi arcs are intimately linked.

\section{Conclusion}

We have shown that ARPES measurements in cuprates can be understood in the context of a Bose-Einstein condensation of preformed pairs. The nature of these composite bosons, pairs of holes in their antiferromagnetic environment, or pairons, naturally imposes a $d$-wave symmetry of the order parameter and gives the correct energy scale of the phase transition, the exchange energy $J$.

Clear Bogoliubov peaks in ARPES and corresponding pseudogap in tunneling reveal the preformed pairs which, due to their strong binding energy, persist in the system at all temperatures below $T^{*}$. Below $T_{\mathrm{c}}$, boson excitations dominate in the antinodal direction (strong pairing) while fermion excitations dominate near the node (weak pairing), giving rise to the Fermi arcs. Above $T_{\mathrm{c}}$, the preformed pairs dissociate continuously, while the Fermi arcs grow at a rate which is doping dependent. Finally, the complete Fermi surface is recovered at $T^{*}$.

In conclusion, the superconducting state, the pseudogap state and the Fermi arcs are tied together as a consequence of a unique phenomenon, the pairons and their excitations.

\section{Acknowledgments}

We gratefully thank $\mathrm{N}$ Miyakawa (Tokyo University of Science), A Fujimori (University of Tokyo) and H Eisaki (AIST Tsukuba) for stimulating discussions.

\section{ORCID iDs}

William Sacks (D) https://orcid.org/0000-0003-1869-7160

\section{References}

[1] Bednorz J G and Müller K A 1986 Possible high $T_{\mathrm{c}}$ superconductivity in the $\mathrm{Ba}-\mathrm{La}-\mathrm{Cu}-\mathrm{O}$ system $Z$. Phys. B 64 189-93

[2] Bardeen J, Cooper L and Schrieffer J 1957 Theory of superconductivity Phys. Rev. 1081175

[3] Fischer Ø, Kugler M, Maggio-Aprile I, Berthod C and Renner C 2007 Scanning tunneling spectroscopy of the cuprates Rev. Mod. Phys. 79353

[4] Damascelli A, Hussain Z and Shen Z-X 2003 Angle-resolved photoemission studies of the cuprate superconductors Rev. Mod. Phys. 75473 
[5] Hashimoto M, Vishik I M, He R-H, Devereaux T P and Shen Z-X 2014 Energy gaps in high-transition-temperature cuprate superconductors Nat. Phys. 10483

[6] Kordyuk A A 2015 Pseudogap from ARPES experiment: three gaps in cuprates and topological superconductivity Low Temp. Phys. $\mathbf{4 1} 319$

[7] Norman M R et al 1998 Destruction of the Fermi surface in underdoped high- $T_{\mathrm{c}}$ superconductors Nature 392 157-60

[8] Timusk T and Statt B 1999 The pseudogap in hightemperature superconductors: an experimental survey Rep. Prog. Phys. 62 61-122

[9] Alloul H, Ohno T and Mendels P $1989^{89} \mathrm{Y}$ NMR evidence for a Fermi-liquid behavior in $\mathrm{YBa}_{2} \mathrm{Cu}_{3} \mathrm{O}_{6+x}$ Phys. Rev. Lett. 631700

[10] Warren W W Jr, Walstedt R E, Brennert G F, Cava R J, Tycko R, Bell R F and Dabbagh G 1989 Cu spin dynamics and superconducting precursor effects in planes above $T_{\mathrm{c}}$ in $\mathrm{YBa}_{2} \mathrm{Cu}_{3} \mathrm{O}_{67}$ Phys. Rev. Lett. 621193

[11] Loram J W, Mirza K A, Wade J M, Cooper J R and Liang W Y 1994 The electronic specific heat of cuprate superconductors Physica C 235-40 134-7

[12] Ding H, Yokoya T, Campuzano J C, Takahashi T, Randeria M, Norman M R, Mochiku T, Kadowaki K and Giapintzakis J 1996 Spectroscopic evidence for a pseudogap in the normal state of underdoped high- $T_{\mathrm{c}}$ superconductors Nature 382 51-4

[13] Loeser A G, Shen Z, Dessau D S, Marshall D S, Park C H, Fournier P and Kapitulnik A 1996 Excitation gap in the normal state of underdoped $\mathrm{Bi}_{2} \mathrm{Sr}_{2} \mathrm{CaCu}_{2} \mathrm{O}_{8+\delta}$ Science 273325

[14] Renner C et al 1998 Pseudogap precursor of the superconducting gap in under- and overdoped $\mathrm{Bi}_{2} \mathrm{Sr}_{2} \mathrm{CaCu}_{2} \mathrm{O}_{8+\delta}$ Phys. Rev. Lett. 80149

[15] Ideta S-I et al 2012 Energy scale directly related to superconductivity in high- $T_{\mathrm{c}}$ cuprates: universality from the temperature-dependent angle-resolved photoemission of $\mathrm{Bi}_{2}$ $\mathrm{Sr}_{2} \mathrm{Ca}_{2} \mathrm{Cu}_{3} \mathrm{O}_{10+d}$ Phys. Rev. B 85104515

[16] Anzai H, Ino A, Arita M, Namatame H, Taniguchi M, Ishikado M W, Fujita K, Ishida S and Uchida S 2013 Relation between the nodal and antinodal gap and critical temperature in superconducting Bi2212 Nat. Commun. 41815

[17] Kaminski A, Kondo T, Takeuchi T and Gu G 2015 Pairing, pseudogap and Fermi arcs in cuprates Phil. Mag. B 95 453-66
[18] Tanaka K, Lee W S, Lu D H, Fujimori A, Fujii T, Terasaki I, Scalapino D J, Devereaux T P, Hussain Z and Shen Z-X 2006 Distinct Fermi-momentum: dependent energy gaps in deeply underdoped Bi2212 Science 3141910

[19] Vishik I M et al 2012 Phase competition in trisected superconducting dome Proc. Natl Acad. Sci. 10918332

[20] Kanigel A, Chatterjee U, Randeria M, Norman M R, Koren G, Kadowaki K and Campuzano J C 2008 Evidence for pairing above the transition temperature of cuprate superconductors from the electronic dispersion in the pseudogap phase Phys. Rev. Lett. 101137002

[21] Shi M et al 2000 Spectroscopic evidence for preformed Cooper pairs in the pseudogap phase of cuprates Eur. Phys. Lett. 8827008

[22] Sacks W, Mauger A and Noat Y 2017 Cooper pairs without 'glue' in high- $T_{\mathrm{c}}$ superconductors: a universal phase diagram Eur. Phys. Lett. 11917001

[23] Poilblanc D, Riera J and Dagotto E 1994 Phys. Rev. B 4912318

[24] Dagotto E 1994 Correlated electrons in high-temperature superconductors Rev. Mod. Phys. 66763

[25] Ando Y, Lavrov A N, Komiya S, Segawa K and Sun X F 2001 Mobility of the doped holes and the antiferromagnetic correlations in underdoped High- $T_{\mathrm{c}}$ cuprates Phys. Rev. Lett. 87017001

[26] Markiewicz R S, Sahrakorpi S, Lindroos M, Lin H and Bansil A 2005 One-band tight-binding model parametrization of the high- $T_{\mathrm{c}}$ cuprates including the effect of $\mathrm{k}_{z}$ dispersion Phys. Rev. B 72054519

[27] Suhl H, Matthias B T and Walker L R 1959 Bardeen-CooperSchrieffer theory of superconductivity in the case of overlapping bands Phys. Rev. Lett. 3552

[28] Sacks W, Mauger A and Noat Y 2015 Pair-pair interactions as a mechanism for high- $T_{\mathrm{c}}$ superconductivity Supercond. Sci. Technol. 28105014

[29] Sacks W, Mauger A and Noat Y 2017 From Cooper-pair glass to unconventional superconductivity: a unified approach to cuprates and pnictides Solid State Commun. 2571

[30] Sacks W, Mauger A and Noat Y 2017 Universal spectral signatures in pnictides, cuprates: the role of quasiparticlepair coupling J. Phys.: Condens. Matter 29445601

[31] Sacks W, Mauger A and Noat Y 2016 Unconventional temperature dependence of the cuprate excitation spectrum Eur. Phys. J. B 89183

[32] Schrieffer J R 1964 Rev. Mod. Phys. 36200 